\title{
On Kauffman bracket skein modules at roots of unity
}

\author{
THANG T Q Lê
}

\begin{abstract}
We reprove and expand results of Bonahon and Wong on central elements of the Kauffman bracket skein modules at roots of 1 and on the existence of the Chebyshev homomorphism, using elementary skein methods.
\end{abstract}

57N10; 57M25

\section{Introduction}

\subsection{Kauffman bracket skein modules}

Let us recall the definition of the Kauffman bracket skein module, which was introduced by J Przytycki [15] and V Turaev [18]. Let $R=\mathbb{C}\left[t^{ \pm 1}\right]$. A framed link in an oriented 3-manifold $M$ is a disjoint union of smoothly embedded circles, equipped with a nonzero normal vector field. The empty set is also considered a framed link. The Kauffman bracket skein module $\mathcal{S}(M)$ is the $R$-module spanned by isotopy classes of framed links in $M$ subject to the relations

$$
\begin{aligned}
L & =t L_{+}+t^{-1} L_{-}, \\
L \sqcup U & =-\left(t^{2}+t^{-2}\right) L,
\end{aligned}
$$

where in the first identity, $L, L_{+}, L_{-}$are identical except in a ball in which they look like they do in Figure 1, and in the second identity, the left-hand side stands for the union of a link $L$ and the trivial framed knot $U$ in a ball disjoint from $L$. If $M=\mathbb{R}^{3}$ then $\mathcal{S}\left(\mathbb{R}^{3}\right)=R$. The value of a framed link $L$ in $\mathcal{S}\left(\mathbb{R}^{3}\right)=R=\mathbb{C}\left[t^{ \pm 1}\right]$ is a version of the Jones polynomial; see Kauffman [10].

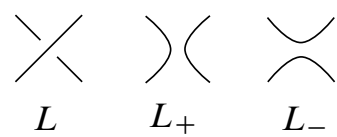

Figure 1: The links $L, L_{+}$and $L_{-}$

For a nonzero complex number $\xi$, let $\mathcal{S}_{\xi}(M)$ be the quotient $\mathcal{S}(M) /(t-\xi)$, which is a $\mathbb{C}$-vector space. 
For an oriented surface $\Sigma$, possibly with boundary, we define $\mathcal{S}(\Sigma):=\mathcal{S}(M)$, where $M=\Sigma \times[-1,1]$ is the cylinder over $\Sigma$. The skein module $\mathcal{S}(\Sigma)$ has an algebra structure induced by the operation of gluing one cylinder on top of the other.

For a framed knot $K$ in $M$ and a polynomial $p(z)=\sum_{j=0}^{d} a_{j} z^{j} \in \mathbb{C}[z]$, then we define $p(K)$ by

$$
p(K)=\sum_{j=0}^{d} a_{j} K^{(j)} \in \mathcal{S}(M),
$$

where $K^{(j)}$ is the link consisting of $j$ parallels of $K$ (using the framing of $K$ ) in a small neighborhood of $K$. When $L$ is a link, define $p(L)$ by applying $p$ to each component of $L$. More precisely, for a framed link $L \subset M$ with $m$ components $L_{1}, \ldots, L_{m}$, define

$$
p(L)=\sum_{j_{1}, \ldots, j_{m}=0}^{d}\left(\prod_{k=1}^{m} a_{j_{k}}\right)\left(\bigsqcup_{k=1}^{m} L_{k}^{\left(j_{k}\right)}\right) .
$$

Here $\bigsqcup_{k=1}^{m} L_{k}^{\left(j_{k}\right)}$ is the link which is the union, over $k \in\{1, \ldots, m\}$, of $j_{k}$ parallels of $L_{k}$.

Remark 0.1 Suppose $K \subset \Sigma$ is a simple closed curve on the surface $\Sigma$. Consider $K$ as a framed knot in $\Sigma \times[-1,1]$ by identifying $\Sigma=\Sigma \times 0$ and equipping $K$ with the vertical framing, ie the framing where the normal vector is perpendicular to $\Sigma$ and has direction from -1 to 1 . Then $K^{(j)}=K^{j}$, where $K^{j}$ is the power in the algebra $\mathcal{S}(\Sigma)$. Thus, $p(K)$ has the usual meaning of applying a polynomial to an element of an algebra.

But if $K$ is a knot in $\Sigma \times[-1,1]$, our $p(K)$ in general is not the result of applying the polynomial $p$ to the element $K$ using the algebra structure of $\mathcal{S}(\Sigma)$, ie $p(K) \neq \sum a_{j} K^{j}$.

\subsection{Bonahon and Wong's results}

Definition 1 A polynomial $p(z) \in \mathbb{C}[z]$ is called central at $\xi \in \mathbb{C}^{\times}$if for any oriented surface $\Sigma$ and any framed link $L$ in $\Sigma \times[-1,1], p(L)$ is central in the algebra $\mathcal{S}_{\xi}(\Sigma)$.

Bonahon and Wong [2] showed that if $\xi$ is a root of unity of order $2 N$, then $T_{N}(z)$ is central, where $T_{N}(z)$ is the Chebyshev polynomial of type 1 defined recursively by

$$
T_{0}(z)=2, \quad T_{1}(z)=1, \quad T_{n}(z)=z T_{n-1}(z)-T_{n-2}(z),
$$

for all $n \geq 2$. We will prove a stronger version, using a different method. 
Theorem 1 A nonconstant polynomial $p(z) \in \mathbb{C}[z]$ is central at $\xi \in \mathbb{C}^{\times}$if and only if

(i) $\xi$ is a root of unity,

(ii) $p(z) \in \mathbb{C}\left[T_{N}(z)\right]$, ie $p$ is a $\mathbb{C}$-polynomial in $T_{N}(z)$, where $N$ is the order of $\xi^{2}$.

Remark 0.2 We also find a version of "skew-centrality" when $\xi^{2 N}=-1$ (see Section 2), which will be useful in this paper and elsewhere.

Remark 0.3 Let us call a polynomial $p(z) \in \mathbb{C}[z]$ weakly central at $\xi \in \mathbb{C}^{\times}$if for any oriented surface $\Sigma$ and any simple closed curve $K$ on $\Sigma, p(K)$ is central in the algebra $\mathcal{S}_{\xi}(\Sigma)$. Then our proof will also show that Theorem 1 holds true if one replaces "central" by "weakly central". It follows that being central is equivalent to being weakly central.

A remarkable result of Bonahon and Wong is the following.

Theorem 2 (Bonahon-Wong [2]) Let $M$ be an oriented 3-manifold, possibly with boundary. Suppose $\xi^{4}$ is a root of unity of order $N$. Let $\varepsilon=\xi^{N^{2}}$. There is a unique $\mathbb{C}$-linear map Ch: $\mathcal{S}_{\varepsilon}(M) \rightarrow \mathcal{S}_{\xi}(M)$ such that for any framed link $L \subset M, \mathbf{C h}(L)=$ $T_{n}(L)$.

If $M=\Sigma \times[-1,1]$, then the map $\mathbf{C h}$ is an algebra homomorphism. Actually Bonahon and Wong only consider the case of $\mathcal{S}(\Sigma)$, but their proof works also in the case of skein modules of 3-manifolds. In their proof, Bonahon and Wong used the theory of quantum Teichmüller space of Chekhov and Fock [7] and Kashaev [9], and the quantum trace homomorphism developed in their earlier work [3]. Bonahon and Wong asked for a proof using elementary skein theory. We will present one here. The main idea is to use central properties (in a more general setting) and several operators and filtrations on the skein modules defined by arcs.

In general, the calculation of $\mathcal{S}(M)$ is difficult. For some results on knot and link complements in $S^{3}$, see the author [11], the author and Tran [12] and Marché [14]. Note that if $\xi^{4 N}=1$, then $\varepsilon=\xi^{N^{2}}$ is a $4^{\text {th }}$ root of 1 . In this case the $\mathcal{S}_{\varepsilon}(M)$ is well known and is related to character varieties of $M$. This makes Theorem 2 interesting. At $t=-1, \mathcal{S}_{-1}(M)$ has an algebra structure and, modulo its nilradical, is equal to the ring of regular functions on the $\mathrm{SL}_{2}(\mathbb{C})$-character variety of $M$; see Bullock [4], Przytycki and Sikora [16] and Bullock, Frohman and Kania-Bartoszyńska [5]. For the case when $\varepsilon$ is a primitive $4^{\text {th }}$ root of 1 , see Sikora [17]. 


\subsection{Plan of the paper}

Section 1 is preliminaries on Chebyshev polynomials and relative skein modules. Section 2 contains the proof of Theorem 1. Section 3 introduces the filtrations and operators on skein modules, and Sections 4 and 5 contain some calculations which are used in Section 6, where the main technical lemma about the skein module of the twice-punctured torus is proved. Theorem 2 is proved in Section 7.

Acknowledgements The author would like to thank F Bonahon, whose talk at the conference "Geometric Topology" at Columbia University (August 12-16, 2013) prompted the author to work on this project. The author also thanks C Frohman, A Sikora and H Wong for helpful discussions. The work is supported in part by the NSF.

\section{Ground ring, Chebyshev polynomials and relative skein modules}

\subsection{Ground ring}

Let $R=\mathbb{C}\left[t^{ \pm 1}\right]$, which is a principal ideal domain. For an $R$-module and a nonzero complex number $\xi \in \mathbb{C}^{\times}$let $V_{\xi}$ be the $R$-module $V /(t-\xi)$. Then $R_{\xi} \cong \mathbb{C}$ as $\mathbb{C}$-modules, and $V_{\xi}$ has a natural structure of an $R_{\xi}$-module.

We will often use the constants

$$
\lambda_{k}:=-\left(t^{2 k+2}+t^{-2 k-2}\right) \in R .
$$

For example, $\lambda_{0}$ is the value of the unknot $U$ as a skein element.

\subsection{Chebyshev polynomials}

Recall that the Chebyshev polynomials of type $1 T_{n}(z)$ and type $2 S_{n}(z)$ are given by

$$
\begin{array}{lll}
T_{0}=2, & T_{1}(z)=z, & T_{n}(z)=z T_{n-1}(z)-T_{n-2}(z), \\
S_{0}=1, & S_{1}(z)=z, & S_{n}(z)=z S_{n-1}(z)-S_{n-2}(z) .
\end{array}
$$

Here are some well-known facts. We drop the easy proofs.

\section{Lemma 1.1 (i) One has}

$$
\begin{aligned}
T_{n}\left(u+u^{-1}\right) & =u^{n}+u^{-n}, \\
T_{n} & =S_{n}-S_{n-2} .
\end{aligned}
$$

(ii) For a fixed positive integer $N$, the $\mathbb{C}-$ span of $\left\{T_{N j} \mid j \geq 0\right\}$ is $\mathbb{C}\left[T_{N}(z)\right]$, the ring of all $\mathbb{C}$-polynomials in $T_{N}(z)$. 
Since $T_{n}(z)$ has leading term $z^{n},\left\{T_{n}(z) \mid n \geq 0\right\}$ is a $\mathbb{C}$-basis of $\mathbb{C}[z]$.

\subsection{Skein module of a surface}

Suppose $\Sigma$ is a compact connected orientable 2-dimensional manifold with boundary. A knot in $\Sigma$ is trivial if it bounds a disk in $\Sigma$. Recall that $\mathcal{S}(\Sigma)$ is the skein module $\mathcal{S}(\Sigma \times[-1,1])$. If $\partial \Sigma \neq \varnothing$, then $\mathcal{S}(\Sigma)$ is a free $R$-module with basis the set of all links in $\Sigma$ without trivial components, including the empty link; see [16]. Here a link in $\Sigma$ is considered as a framed link in $\Sigma \times[-1,1]$ by identifying $\Sigma$ with $\Sigma \times 0$, and the framing at every point $P \in \Sigma \times 0$ is vertical, ie given by the unit positive tangent vector of $P \times[-1,1] \subset \Sigma \times[-1,1]$.

The $R$-module $\mathcal{S}(\Sigma)$ has a natural $R$-algebra structure, where $L_{1} L_{2}$ is obtained by placing $L_{1}$ on top of $L_{2}$.

It might happen that $\Sigma_{1} \times[-1,1] \cong \Sigma_{2} \times[-1,1]$ with $\Sigma_{1} \not \Sigma_{2}$. In that case, $\mathcal{S}\left(\Sigma_{1}\right)$ and $\mathcal{S}\left(\Sigma_{2}\right)$ are the same as $R$-modules, but the algebra structures may be different.

\subsection{Example: The annulus}

Let $\mathbb{A} \subset \mathbb{R}^{2}$ be the annulus $\mathbb{A}=\left\{\vec{x} \in \mathbb{R}^{2}|1 \leq| \vec{x} \mid \leq 2\right\}$. Let $z \in \mathcal{S}(\mathbb{A})$ be the core of the annulus, $z=\left\{\vec{x},|\vec{x}|=\frac{3}{2}\right\}$. Then $\mathcal{S}(\mathbb{A})=R[z]$.

\subsection{Relative skein modules}

A marked surface $(\Sigma, \mathcal{P})$ is a surface $\Sigma$ together with a finite set $\mathcal{P}$ of points on its boundary $\partial \Sigma$. For such a marked surface, a relative framed link is a 1-dimensional compact framed submanifold $X$ in $\Sigma \times[-1,1]$ such that $\partial X=\mathcal{P}=X \cap \partial(\Sigma \times[-1,1]), X$ is perpendicular to $\partial(\Sigma \times[-1,1])$ and the framing at each point $P \in \mathcal{P}=\partial X$ is vertical. The relative skein module $\mathcal{S}(\Sigma, \mathcal{P})$ is defined as the $R$-module spanned by the isotopy class of relative framed links modulo the same skein relations (1) and (2). We will use the following fact.

Proposition 1.2 [16, Theorem 5.2] The $R$-module $\mathcal{S}(\Sigma, \mathcal{P})$ is free with basis the set of isotopy classes of relative links embedded in $\Sigma$ without trivial components.

\section{Annulus with two marked points and central elements}

\subsection{Marked annulus}

Recall that $\mathbb{A} \subset \mathbb{R}^{2}$ is the annulus $\mathbb{A}=\left\{\vec{x} \in \mathbb{R}^{2}|1 \leq| \vec{x} \mid \leq 2\right\}$. Let $\mathbb{A}_{i o}$ be the marked surface $\left(\mathbb{A},\left\{P_{1}, P_{2}\right\}\right)$, with two marked points $P_{1}=(0,1), P_{2}=(0,2)$, which are on different boundary components. See Figure 2 , which also depicts the $\operatorname{arcs} e, \mathfrak{u}, \mathfrak{u}^{-1}$. 


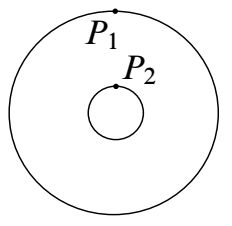

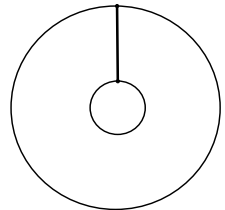

$\operatorname{arc} e$

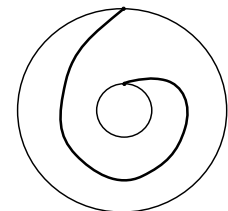

$\operatorname{arc} u$

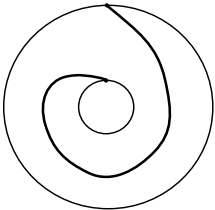

$\operatorname{arc} u^{-1}$

Figure 2: The marked annulus $\mathbb{A}_{i o}$ and the $\operatorname{arcs} e, \mathfrak{u}$ and $\mathfrak{u}^{-1}$

For $L_{1}, L_{2} \in \mathcal{S}\left(\mathbb{A}_{i o}\right)$ define the product $L_{1} L_{2}$ by placing $L_{1}$ inside $L_{2}$. Formally this means we first shrink $\mathbb{A}_{i o} \supset L_{1}$ by $\frac{1}{2}$, we get $\left(\frac{1}{2} \mathbb{A}_{i o}\right) \supset\left(\frac{1}{2} L_{1}\right)$, where $\frac{1}{2} \mathbb{A}_{i o}$ is an annulus on the plane whose outer circle is the inner circle of $\mathbb{A}_{i o}$. Then $L_{1} L_{2}$ is $\left(\frac{1}{2} L_{1}\right) \cup L_{2} \subset\left(\frac{1}{2} \mathbb{A}_{i o}\right) \cup \mathbb{A}_{i o}$. The identity of $\mathcal{S}\left(\mathbb{A}_{i o}\right)$ is presented by $e$, and $\mathfrak{u}^{-1} \mathfrak{u}=$ $e=\mathfrak{u u}^{-1}$.

Proposition 2.1 The Kauffman bracket skein modules of $\mathbb{A}_{i o}$ are $\mathcal{S}\left(\mathbb{A}_{i o}\right)=R\left[\mathfrak{u}^{ \pm 1}\right]$, the ring of Laurent $R$-polynomials in one variable $\mathfrak{u}$. In particular, $\mathcal{S}\left(\mathbb{A}_{i o}\right)$ is commutative.

Proof Using Proposition 1.2 one can easily show that the set $\left\{\mathfrak{u}^{k} \mid k \in \mathbb{Z}\right\}$ is a free $R$-basis of $\mathcal{S}\left(\mathbb{A}_{i o}\right)$.

\subsection{Passing through $T_{k}$}

Recall that $\mathcal{S}(\mathbb{A})=R[z]$. One defines a left action and a right action of $\mathcal{S}(\mathbb{A})$ on $\mathcal{S}\left(\mathbb{A}_{i o}\right)$ as follows. For $L \in \mathcal{S}(\mathbb{A}), K \in \mathcal{S}\left(\mathbb{A}_{i o}\right)$ let $L \bullet K$ be the element in $\mathcal{S}\left(\mathbb{A}_{i o}\right)$ obtained by placing $L$ above $K$, and $K \bullet L \in \mathcal{S}\left(\mathbb{A}_{i o}\right)$ be the element in $\mathcal{S}\left(\mathbb{A}_{i o}\right)$ obtained by placing $K$ above $L$. For example,

$$
e \cdot z=(0), \quad z \bullet e=(0) \text {. }
$$

Proposition 2.2 One has

$$
\begin{aligned}
T_{k}(z) \bullet e & =t^{k} \mathfrak{u}^{k}+t^{-k} \mathfrak{u}^{-k}, \\
e \bullet T_{k}(z) & =t^{k} \mathfrak{u}^{-k}+t^{-k} \mathfrak{u}^{k}, \\
T_{k}(z) \bullet e-e \bullet T_{k}(z) & =\left(t^{k}-t^{-k}\right)\left(\mathfrak{u}^{k}-\mathfrak{u}^{-k}\right) .
\end{aligned}
$$

Proof It is important to note that the map $f: \mathcal{S}(\mathbb{A}) \rightarrow \mathcal{S}\left(\mathbb{A}_{\text {io }}\right)$ given by $f(L)=L \bullet e$ is an algebra homomorphism. 
Resolve the only crossing point, we have

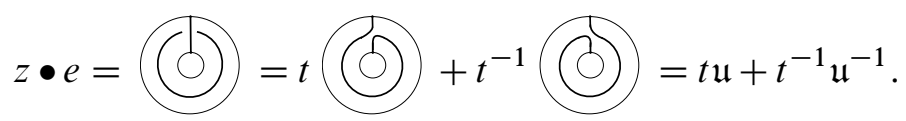

Hence

$$
\begin{aligned}
T_{k}(z) \bullet e & =T_{k}\left(t \mathfrak{u}+t^{-1} \mathfrak{u}^{-1}\right) & & \text { (because } f \text { is an algebra homomorphism) } \\
& =t^{k} \mathfrak{u}^{k}+t^{-k} \mathfrak{u}^{-k} & & \text { (by (4)). }
\end{aligned}
$$

This proves (6). The proof of (7) is similar, while (8) follows from (6) and (7).

Corollary 2.3 Suppose $\xi^{2 N}=1$. Then $T_{N}(z)$ is central at $\xi$.

Proof We have $\xi^{N}=\xi^{-N}$ since $\xi^{2 N}=1$. Then (8) shows that $T_{N}(z) \bullet e=e \bullet T_{N}(z)$, which easily implies the centrality of $T_{N}(z)$.

Remark 2.4 The corollary was first proved by Bonahon and Wong [2] using another method.

\subsection{Transparent elements}

We say that $p(z) \in \mathbb{C}[z]$ is transparent at $\xi$ if for any 3 disjoint framed knots $K, K_{1}, K_{2}$ in any oriented 3-manifold $M, p(K) \cup K_{1}=p(K) \cup K_{2}$ in $\mathcal{S}_{\xi}(M)$, provided that $K_{1}$ and $K_{2}$ are isotopic in $M$. Note that in general, $K_{1}$ and $K_{2}$ are not isotopic in $M \backslash K$.

Proposition 2.5 The following are equivalent.

(i) $p(z) \bullet e=e \bullet p(z)$ in $\mathcal{S}_{\xi}\left(\mathbb{A}_{i o}\right)$.

(ii) $p(z)$ is transparent at $\xi$.

(iii) $p(z)$ is central at $\xi$.

Proof It is clear that (i) $\Rightarrow$ (ii) $\Rightarrow$ (iii). Let us prove (iii) $\Rightarrow$ (i).

By gluing a 1-handle to $\mathbb{A}$ we get a punctured torus $\mathbb{T}_{\text {punc }}$ as in Figure 3 . Here the base of the 1-handle is glued to a small neighborhood of $\left\{P_{1} \cup P_{2}\right\}$ in $\partial \mathbb{A}$, and the core of the 1 -handle is an arc $\beta$ connecting $P_{1}$ and $P_{2}$. Let $\iota: \mathcal{S}\left(\mathbb{A}_{i o}\right) \rightarrow \mathcal{S}\left(\mathbb{T}_{\text {punc }}\right)$ be the $R$-map which is the closure by $\beta$, ie $\iota(K)=K \cup \beta$. Then $\iota\left(\mathfrak{u}^{k}\right)$ is a knot in $\mathbb{T}_{\text {punc }}$ for every $k \in \mathbb{Z}$, and $\iota\left(\mathfrak{u}^{k}\right)$ is not isotopic to $\iota\left(\mathfrak{u}^{l}\right)$ if $k \neq l$. Since $\left\{\mathfrak{u}^{k} \mid k \in \mathbb{Z}\right\}$ is an $R$-basis of $\mathcal{S}\left(\mathbb{A}_{i o}\right)$ and the isotopy classes of links in $\mathbb{T}_{\text {punc }}$ form an $R$-basis of $\mathcal{S}\left(\mathbb{T}_{\text {punc }}\right), \iota$ is injective.

Assume (iii). Then $p(z) \iota(e)=\iota(e) p(z)$, or $\iota(p(z) \bullet e)=\iota(e \bullet p(z))$. Since $\iota$ is injective, we have $p(z) \bullet e=e \bullet p(z)$. 


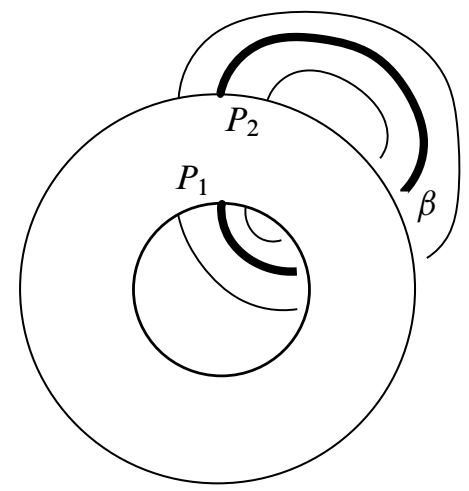

Figure 3: The core $\beta$ connects $P_{1}$ and $P_{2}$ in $\mathbb{T}_{\text {punc }}$.

\subsection{Proof of Theorem 1}

The "if" part has been proved; see Corollary 2.3. Let us prove the "only if" part. Assume that $p(z)$ is central at $\xi$ and has degree $k \geq 1$. Since $\left\{T_{j}(z) \mid j \geq 0\right\}$ is a basis of $\mathbb{C}[z]$, we can write

$$
p(z)=\sum_{j=0}^{k} c_{j} T_{j}(z), \quad c_{j} \in \mathbb{C}, c_{k} \neq 0 .
$$

By Proposition 2.5, $p(z) \bullet e-e \bullet p(z)=0$. Using expression (9) for $p(z)$ and (8), we get

$$
0=p(z) \bullet e-e \bullet p(z)=\sum_{j=0}^{k} c_{j}\left(\xi^{j}-\xi^{-j}\right)\left(\mathfrak{u}^{j}-\mathfrak{u}^{-j}\right) .
$$

Because $\left\{\mathfrak{u}^{j} \mid j \in \mathbb{Z}\right\}$ is a basis of $\mathcal{S}_{\xi}\left(\mathbb{A}_{i o}\right)$, the coefficient of each $\mathfrak{u}^{j}$ on the right-hand side is 0 . This means

$$
c_{j}=0 \quad \text { or } \xi^{2 j}=1 \text { for all } j .
$$

Since $c_{k} \neq 0$, we have $\xi^{2 k}=1$. Since $k \geq 1$, this shows $\xi^{2}$ is a root of unity of some order $N$. Then (10) shows that $c_{j}=0$ unless $N \mid j$. Thus, $p(z)$ is a $\mathbb{C}$-linear combination of $T_{j}$ with $N \mid j$. This completes the proof of Theorem 1.

\subsection{Skew transparency}

One more consequence of Proposition 2.2 is the following.

Corollary 2.6 Suppose $\xi^{2 N}=-1$. Then in $\mathcal{S}_{\xi}\left(\mathbb{A}_{i o}\right)$,

$$
T_{N}(z) \bullet e=-e \bullet T_{N}(z) \text {. }
$$


This means every time we pass $T_{N}(K)$ through a component of a link $L$, the value of the skein gets multiplied by -1 . Following is a precise statement.

Suppose $K_{1}$ and $K_{2}$ are knots in a 3-manifold $M$. Recall that an isotopy between $K_{1}$ and $K_{2}$ is a smooth map $H: S^{1} \times[1,2] \rightarrow M$ such that for each $t \in[1,2]$, the map $H_{t}: S^{1} \rightarrow M$ is an embedding, and the image of $H_{i}$ is $K_{i}$ for $i=1,2$. Here $H_{t}(x)=H(x, t)$. For a knot $K \subset M$ let $I_{2}(H, K)$ be the mod 2 intersection number of $H$ and $K$. Thus, if $H$ is transversal to $K$ then $I_{2}(H, K)$ is the number of points in the finite set $H^{-1}(K)$ modulo 2 .

Definition 2 Suppose $\mu= \pm 1$. A polynomial $p(z) \in \mathbb{C}[z]$ is called $\mu$-transparent at $\xi \in \mathbb{C}^{\times}$if for any 3 disjoint framed knots $K, K_{1}, K_{2}$ in any oriented 3-manifold $M$, with $K_{1}$ and $K_{2}$ connected by an isotopy $H$, one has the following equality in $\mathcal{S}_{\xi}(M)$ :

$$
p(K) \cup K_{1}=\mu^{I_{2}(H, K)}\left[p(K) \cup K_{2}\right] .
$$

From Corollary 2.6 we have:

Corollary 2.7 Assume $\xi^{4 N}=1$. Then $\mu:=\xi^{2 N}= \pm 1$, and $T_{N}(z)$ is $\mu$-transparent.

A special case is the following. Suppose $D \subset M$ is a disk in $M$ with $\partial D=K$, and a framed link $L \subset M$ is disjoint from $K$. Then, if $\xi^{2 N}=\mu= \pm 1$, one has

$$
K \cup T_{N}(L)=\mu^{I_{2}(D, L)} \lambda_{0} T_{N}(L) \text { in } \mathcal{S}_{\xi}(M) .
$$

Here $\lambda_{0}=-\left(\xi^{2}+\xi^{-2}\right)$ is the value of trivial knot in $\mathcal{S}_{\xi}(M)$.

\section{Filtrations of skein modules}

Suppose $\Phi$ is a link in $\partial M$. We define an $R$-map $\Phi: \mathcal{S}(M) \rightarrow \mathcal{S}(M)$ by $\Phi(L)=$ $\Phi \cup L$.

\subsection{Filtration by an arc}

Suppose $\alpha$ is an arc properly embedded in a marked surface $(\Sigma, \mathcal{P})$ with $\partial \Sigma \neq \varnothing$. Assume the two boundary points of $\alpha$, which are on the boundary of $\Sigma$, are disjoint from the marked points. Then $\mathcal{D}_{\alpha}:=\alpha \times[-1,1]$ is a disk properly embedded in $\Sigma \times[-1,1]$, with boundary $\Phi_{\alpha}=\partial(\alpha \times[-1,1])=(\alpha \times\{-1,1\}) \cup(\partial \alpha \times[-1,1])$.

Let $\mathcal{F}_{k}^{\alpha}=\mathcal{F}_{k}^{\alpha}(\mathcal{S}(\Sigma))$ be the $R$-submodule of $\mathcal{S}(\Sigma)$ spanned by all relative links which intersect with $\mathcal{D}_{\alpha}$ at less than or equal to $k$ points. For $L \in \mathcal{S}(\Sigma)$, we define fil $_{\alpha}(L)=k$ if $L \in \mathcal{F}_{k}^{\alpha} \backslash \mathcal{F}_{k-1}^{\alpha}$. The filtration is compatible with the algebra structure, ie

$$
\operatorname{fil}_{\alpha}\left(L_{1} L_{2}\right) \leq \operatorname{fil}_{\alpha}\left(L_{1}\right)+\operatorname{fil}_{\alpha}\left(L_{2}\right) .
$$


Remark 3.1 A similar filtration was used in [14] to calculate the skein module of torus knot complements.

A convenient way to count the number of intersection points of a link $L$ with $\mathcal{D}_{\alpha}$ is to count the intersection points of the diagram of $L$ with $\alpha$. Let $D$ be the vertical projection of $L$ onto $\Sigma$. In general position $D$ has only singular points of type double points, and we assume further that $D$ is transversal to $\alpha$. In that case, the number of intersection points of $L$ with $\mathcal{D}_{\alpha}$ is equal to the number of intersections of $D$ with $\alpha$, where each intersection point of $\alpha$ and $D$ at a double point of $D$ is counted twice.

Recall that $\Phi_{\alpha}(L)=L \cup \Phi_{\alpha}$, where $\Phi_{\alpha}$ is the boundary of the disk $\mathcal{D}_{\alpha}=\alpha \times[-1,1]$. It is clear that $\mathcal{F}_{k}^{\alpha}$ is $\Phi_{\alpha}$-invariant, ie $\Phi_{\alpha}\left(\mathcal{F}_{k}^{\alpha}\right) \subset \mathcal{F}_{k}^{\alpha}$. It turns out that the action of $\Phi_{\alpha}$ on the quotient $\mathcal{F}_{k}^{\alpha} / \mathcal{F}_{k-1}^{\alpha}$ is very simple. Recall that $\lambda_{k}=-\left(t^{2 k+2}+t^{-2 k-2}\right)$.

Proposition 3.2 For $k \geq 0$, the action of $\Phi_{\alpha}$ on $\mathcal{F}_{k}^{\alpha} / \mathcal{F}_{k-1}^{\alpha}$ is $\lambda_{k}$ times the identity.

This is a consequence of Proposition 3.3, proved in the next subsection.

\subsection{The Temperley-Lieb algebra and the operator $\Phi$}

The well-known Temperley-Lieb algebra $\mathrm{TL}_{k}$ is the skein module of the disk with $2 k$ marked points on the boundary. We will present the disk as the square $\mathrm{Sq}=[0,1] \times[0,1]$ on the standard plane, with $k$ marked points on the top side and $k$ marked points on the bottom side. The product $L_{1} L_{2}$ in $\mathrm{TL}_{k}$ is defined as the result of placing $T_{1}$ on top of $T_{2}$. The unit $\widetilde{e}_{k}$ of $\mathrm{TL}_{k}$ is presented by $k$ vertical straight arcs; see Figure 4.

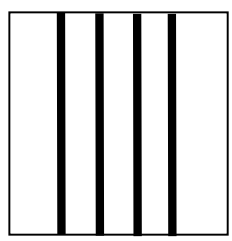

$\tilde{e}_{k}$

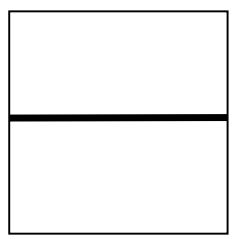

$\operatorname{arc} \alpha$

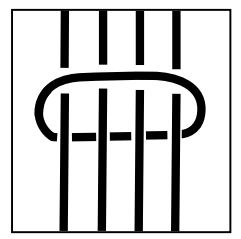

$\Phi_{\alpha}\left(\widetilde{e}_{k}\right)$

Figure 4: The unit $\widetilde{e}_{k}$, the arc $\alpha$ and $\Phi_{\alpha}\left(\widetilde{e}_{k}\right)$ : here $k=4$

Let $\alpha \subset$ Sq be the horizontal arc $[0,1] \times \frac{1}{2}$. The element $\Phi_{\alpha}\left(\widetilde{e}_{k}\right)$ is depicted in Figure 4 . In general, $\Phi_{\alpha}(L)$ is $L$ encircled by one simple closed curve.

Proposition 3.3 With the above notation, one has

$$
\Phi_{\alpha}\left(\widetilde{e}_{k}\right)=\lambda_{k} \widetilde{e}_{k}\left(\bmod \mathcal{F}_{k-1}^{\alpha}\right) .
$$


Proof A direct proof can be carried out as follows. Using the skein relation (1) one resolves all the crossings of the diagram of $\Phi_{\alpha}\left(\widetilde{e}_{k}\right)$, and finds that only a few terms are not in $\mathcal{F}_{k-1}^{\alpha}$, and the sum of these terms is equal to $\lambda_{k} \widetilde{e}_{k}$. This is a good exercise for the dedicated reader.

Here is another proof using more advanced knowledge of the Temperley-Lieb algebra. First we extend the ground ring to the field of fractions $\mathbb{C}(t)$. Then the Temperley-Lieb algebra contains a special element called the Jones-Wenzl idempotent $f_{k}$ (see eg Lickorish [13, Chapter 13]). We have $f_{k}=\widetilde{e}_{k}\left(\bmod \mathcal{F}_{k-1}^{\alpha}\right)$, and $f_{k}$ is an eigenvector of $\Phi_{\alpha}$ with eigenvalue $\lambda_{k}$. Hence, we have (12).

\section{Another annulus with two marked points}

\subsection{Annulus with two marked points on the same boundary}

Let $\mathbb{A}_{\text {oo }}$ be the annulus $A$ with two marked points $Q_{1}, Q_{2}$ on the outer boundary as in Figure 5. Let $u_{0}, u_{1}$ be arcs connecting $Q_{1}$ and $Q_{2}$ in $\mathbb{A}_{o o}$ as in Figure 5.
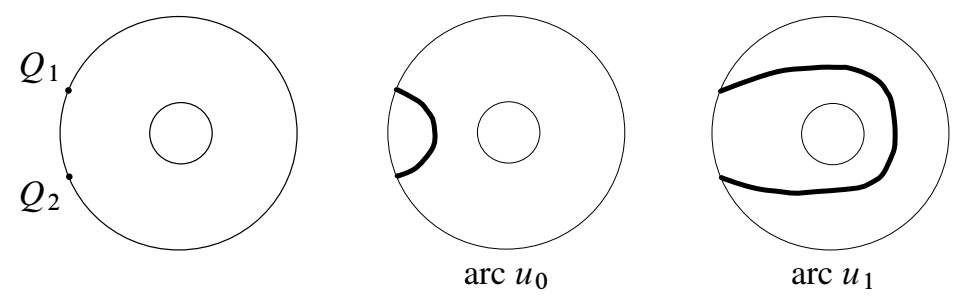

Figure 5: The marked annulus $\mathbb{A}_{\text {oo }}$ and $\operatorname{arcs} u_{0}, u_{1}$

Define a left $\mathcal{S}(\mathbb{A})$-module and a right $\mathcal{S}(\mathbb{A})$-module on $\mathcal{S}\left(\mathbb{A}_{\text {oo }}\right)$ as follows. For $K \in \mathcal{S}\left(\mathbb{A}_{o o}\right)$ and $L \in \mathcal{S}(\mathbb{A})$ let $K L$ be the skein in $\mathcal{S}\left(\mathbb{A}_{o o}\right)$ obtained by placing $K$ on top of $L$, and $L K \in \mathcal{S}\left(\mathbb{A}_{o o}\right)$ obtained by placing $L$ on top of $K$. It is easy to see that $K L=L K$. Recall that $\mathcal{S}(\mathbb{A})=R[z]$.

Proposition 4.1 The module $\mathcal{S}\left(\mathbb{A}_{o o}\right)$ is a free $\mathcal{S}(\mathbb{A})$-module with basis $\left\{u_{0}, u_{1}\right\}$ :

$$
\mathcal{S}\left(\mathbb{A}_{\text {oo }}\right)=R[z] u_{0} \oplus R[z] u_{1} .
$$

Proof Any relative link in $\mathbb{A}_{o o}$ is of the form $u_{i} z^{m}$ with $i=0,1$ and $m \in \mathbb{Z}$. The proposition now follows from Proposition 1.2. 


\subsection{Framing change and the unknot}

Recall that $S_{k}$ is the $k^{\text {th }}$ Chebyshev polynomial of type 2 . The values of the unknot colored by $S_{k}$ and the framing change are well known (see eg Blanchet, Habegger, Masbaum and Vogel [1]): in $\mathcal{S}(M)$, where $M$ is an oriented 3-manifold, one has

$$
\begin{aligned}
& L \sqcup S_{k}(U)=(-1)^{k} \frac{t^{2 k+2}-t^{-2 k-2}}{t^{2}-t^{-2}} L, \\
& S_{k}(\bigcirc)=(-1)^{k} t^{k^{2}+2 k} S_{k}(\mid) .
\end{aligned}
$$

Here in (13), $U$ is the trivial knot lying in a ball disjoint from $L$.

\subsection{Some elements of $\mathcal{S}\left(\mathbb{A}_{\text {oo }}\right)$}

Let $u_{k}, k \geq 0$ are arcs in $\mathbb{A}_{\text {oo }}$ depicted in Figure 6. The elements $u_{1}$ and $u_{0}$ are the same as the ones defined in Figure 5. Let $v_{0}=u_{0}$ and $v_{k}, k \geq 1$ be $\operatorname{arcs}$ in $\mathbb{A}_{\text {oo }}$ depicted in Figure 6.
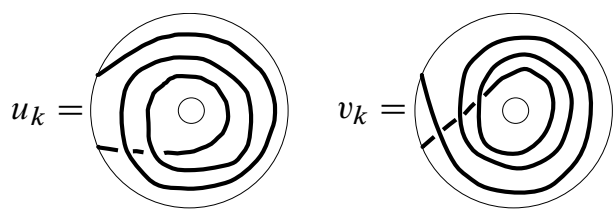

Figure 6: The arcs $u_{k}$ and $v_{k}$, with $k=3$

Proposition 4.2 One has

$$
\begin{aligned}
& u_{k}=t^{k-1} S_{k-1}(z) u_{1}+t^{k-3} S_{k-2}(z) u_{0}, \\
& v_{k}=t^{2-k} S_{k-1}(z) u_{1}+t^{-k} S_{k}(z) u_{0},
\end{aligned}
$$

for all $k \geq 1$, for all $k \geq 0$, respectively.

Proof Suppose $k \geq 3$. Applying the skein relation to the innermost crossing of $u_{k}$, we get

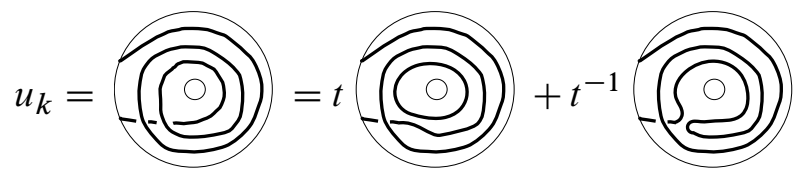

which, after an isotopy and removing a framing crossing, is

$$
u_{k}=t u_{k-1} z-t^{2} u_{k-2} \text {, }
$$

from which one can easily prove (15) by induction. 
Similarly, using the skein relation to resolve the innermost crossing point of $v_{k}$, we get

$$
v_{k}=t^{-1} v_{k-1} z-t^{-2} v_{k-2} \text { for } k \geq 2,
$$

from which one can prove (16) by induction.

Remark 4.3 Identity (15) does not hold for $k=0$. This is due to a framing change.

\subsection{Operator $\Psi$}

Let $\Psi$ be the arc in $\partial \mathbb{A} \times[-1,1]$ beginning at $Q_{1}$ and ending at $Q_{2}$, as depicted in Figure 7. Here we draw $\mathbb{A} \times[-1,1]$ as a handlebody. For any element $\alpha \in \mathcal{S}(\mathbb{A})$ let

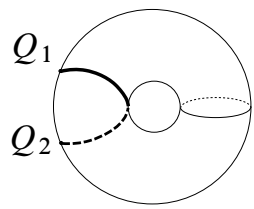

$\operatorname{arc} \Psi$

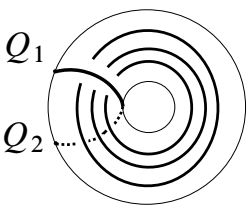

$\Psi\left(z^{3}\right)$

Figure 7: Arc $\Psi$ connecting $Q_{1}$ and $Q_{2}$ and $\Psi\left(z^{3}\right)$

$\Psi(\alpha) \in \mathcal{S}\left(\mathbb{A}_{o o}\right)$ be the skein $\Psi \cup \alpha$. For example, $\Psi\left(z^{3}\right)$ is given in Figure 7.

Proposition 4.4 For $k \geq 1$, one has

$$
\Psi\left(T_{k}(z)\right)=u_{1}\left[t^{2}\left(t^{-2 k}-t^{2 k}\right) S_{k-1}(z)\right]+u_{0}\left[t^{-2 k} S_{k}(z)-t^{2 k} S_{k-2}(z)\right] .
$$

Proof Applying Proposition 2.2 to the part in the left rectangle box, we get

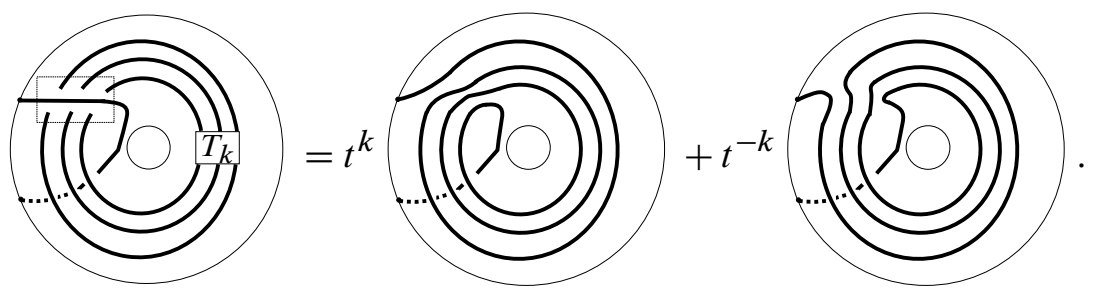

The positive framing crossing in the first term gives a factor $-t^{3}$. Thus

$$
\Psi\left(T_{k}(z)\right)=-t^{k+3} u_{k}+t^{-k} v_{k} .
$$

Plugging in the values of $u_{k}, v_{k}$ given by Proposition 4.2, we get the result.

Remark 4.5 One can use Proposition 4.4 to establish product-to-sum formulas similar to the ones in Frohman and Gelca [8]. 


\section{Twice-punctured disk}

\subsection{Skein module of twice-punctured disk}

Let $\mathcal{D} \subset \mathbb{R}^{2}$ be the disk of radius 4 centered at the origin, $\mathcal{D}_{1} \subset \mathbb{R}^{2}$ the disk of radius 1 centered at $(-2,0)$, and $\mathcal{D}_{2}$ the disk of radius 1 centered at $(2,0)$. We define $\mathbb{D}$ to
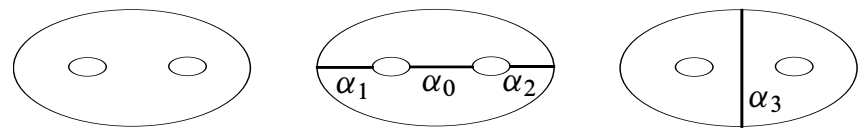

Figure 8: The twice-punctured disk $\mathbb{D}$ and the $\operatorname{arcs} \alpha_{1}, \alpha_{0}, \alpha_{2}, \alpha_{3}$

be $\mathcal{D}$ with the interiors of $\mathcal{D}_{1}$ and $\mathcal{D}_{2}$ removed. The horizontal axis intersects $\mathbb{D}$ at 3 arcs denoted from left to right by $\alpha_{1}, \alpha_{0}, \alpha_{2}$; see Figure 8 . The vertical axis of $\mathbb{R}^{2}$ intersects $\mathbb{D}$ at an arc denoted by $\alpha_{3}$. The corresponding curve $\Phi_{\alpha_{i}}$ on $\partial \mathbb{D} \times[-1,1]$ will be denoted simply by $\Phi_{i}$ for $i=0,1,2,3$. If $\mathbb{D} \times[-1,1]$ is presented as the handlebody $\mathcal{H}$, which is a thickening of $\mathbb{D}$ in $\mathbb{R}^{3}$, then the curves $\Phi_{1}, \Phi_{0}, \Phi_{2}, \Phi_{3}$ are shown in Figure 9.
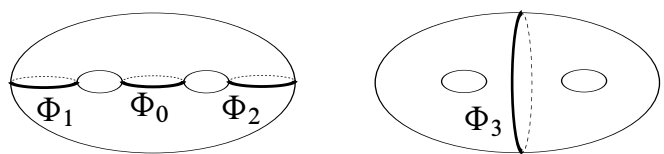

Figure 9: The curves $\Phi_{1}, \Phi_{0}, \Phi_{2}, \Phi_{3}$ on the boundary of the handlebody

Let $x_{1}, x_{2}$, and $y$ be the closed curves in $\mathbb{D}$ :

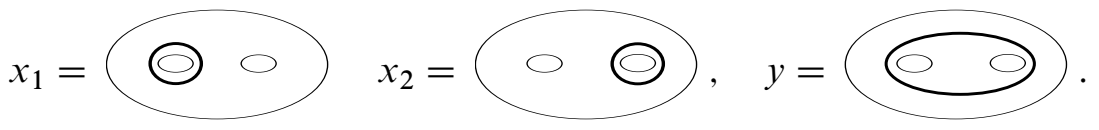

It is known that we have the equality $\mathcal{S}(\mathbb{D})=R\left[x_{1}, x_{2}, y\right]$, the $R$-polynomial in the variables $x_{1}, x_{2}, y$; see Bullock and Przytycki [6]. In particular, $\mathcal{S}(\mathbb{D})$ is commutative. Let $\sigma$ be the rotation about the origin of $\mathbb{R}^{2}$ by $180^{\circ}$. Then $\sigma(\mathbb{D})=\mathbb{D}$. Hence $\sigma$ induces an automorphism of $\mathcal{S}(\mathbb{D})=R\left[x_{1}, x_{2}, y\right]$, which is an algebra automorphism. One has $\sigma(y)=y, \sigma\left(x_{1}\right)=x_{2}, \sigma\left(x_{2}\right)=x_{1}$.

\subsection{Degrees on $\mathcal{S}(\mathbb{D})=R\left[x_{1}, x_{2}, y\right]$}

Define the left degree, right degree and double degree on $R\left[x_{1}, y, x_{2}\right]$ as follows. For a monomial $\mathfrak{m}=x_{1}^{a_{1}} y^{b} x_{2}^{a_{2}}$ define its left degree $\operatorname{deg}_{l}(\mathfrak{m})=a_{1}+b$, right degree 
$\operatorname{deg}_{r}(\mathfrak{m})=a_{2}+b$, double degree $\operatorname{deg}_{l r}(\mathfrak{m})=\operatorname{deg}_{l}(\mathfrak{m})+\operatorname{deg}_{r}(\mathfrak{m})=a_{1}+a_{2}+2 b$. One readily finds that

$$
\operatorname{deg}_{l}(\mathfrak{m})=\operatorname{fil}_{\alpha_{1}}(\mathfrak{m}), \quad \operatorname{deg}_{r}(\mathfrak{m})=\operatorname{fil}_{\alpha_{2}}(\mathfrak{m}),
$$

where $\mathrm{fil}_{\alpha}$ is defined in Section 3.1. Using the definition of fil $\alpha$ involving the numbers of intersection points we get the following.

Lemma 5.1 Suppose $L$ is an embedded link in $\mathbb{D}$ and $L$ intersects transversally the arc $\alpha_{i}$ at $k_{i}$ points for $i=1,2,3$. Then, as an element of $\mathcal{S}(\mathbb{D}), L=x_{1}^{a_{1}} x_{2}^{a_{2}} y^{b}$, where $2 b \leq k_{3}$ and

$$
\begin{array}{ll}
\operatorname{deg}_{l}(L) \leq k_{1}, & \operatorname{deg}_{l}(L) \equiv k_{1}(\bmod 2), \\
\operatorname{deg}_{r}(L) \leq k_{2}, & \operatorname{deg}_{r}(L) \equiv k_{2}(\bmod 2) .
\end{array}
$$

Consequently, $\operatorname{deg}_{l r}(L) \leq k_{1}+k_{2}$ and $\operatorname{deg}_{l r}(L) \equiv k_{1}+k_{2}(\bmod 2)$.

Proof If $L=L_{1} \sqcup L_{2}$ is the union of 2 disjoint sublinks, and the statement holds for each of $L_{i}$, then it holds for $L$. Hence we assume $L$ has one component, ie $L$ is an embedded loop in $\mathbb{D} \subset \mathbb{R}^{2}$. Then $L$ is isotopic to either a trivial loop, $x_{1}, x_{2}$ or $y$. In each case, the statement can be verified easily. For example, suppose $L=x_{1}$. For the mod 2 intersection numbers, $I_{2}\left(L, \alpha_{1}\right)=I_{2}\left(x_{1}, \alpha_{1}\right)=1$. Hence $k_{1}$, the geometric intersection number between $L$ and $\alpha_{1}$, must be odd and bigger than or equal to 1 . Hence, we have $\operatorname{deg}_{l}(L) \leq k_{1}$ and $\operatorname{deg}_{l}(L) \equiv k_{1}(\bmod 2)$.

Corollary 5.2 Suppose $L$ is a link diagram on $\mathbb{D}$ which intersects transversally the $\operatorname{arc} \alpha_{i}$ at $k_{i}$ points for $i=1,2,3$. Then, as an element in $\mathcal{S}(\mathbb{D})$,

$$
\operatorname{deg}_{l}(L) \leq k_{1}, \quad \operatorname{deg}_{r}(L) \leq k_{2}, \quad 2 \operatorname{deg}_{y}(L) \leq k_{3},
$$

and $L$ is a linear $R$-combination of monomials whose double degrees are equal to $k_{1}+k_{2}$ modulo 2 .

\subsection{The $R$-module $V_{n}$ and the skein $\gamma$}

Let $\gamma$ and $\bar{\gamma}$ be the following link diagrams on $\mathbb{D}$ :

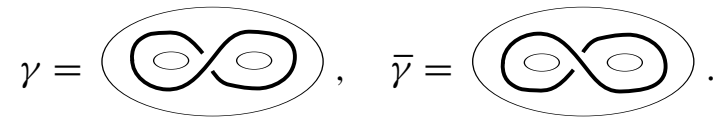

Let

$$
V_{n}=\left\{p \in R\left[x_{1}, x_{2}, y\right] \mid \operatorname{deg}_{l}(p) \leq n, \operatorname{deg}_{r}(p) \leq n, \operatorname{deg}_{l r}(p) \text { even }\right\} .
$$

In other words, $V_{n} \subset R\left[x_{1}, x_{2}, y\right]$ is the $R$-submodule spanned by $x_{1}^{a_{1}} x_{2}^{a_{2}} y^{b}$, with $a_{i}+b \leq n$ for $i=1,2$ and $a_{1}+a_{2}$ even. 
Lemma 5.3 One has $T_{n}(\gamma), T_{n}(\bar{\gamma}) \in V_{n}$.

Proof The diagram $\gamma^{k}$ has $k$ intersection points with each of $\alpha_{1}$ and $\alpha_{2}$. By Corollary 5.2, we have $\operatorname{deg}_{l}\left(\gamma^{k}\right) \leq k, \operatorname{deg}_{r}\left(\gamma^{k}\right) \leq k$, and each monomial of $\gamma^{k}$ has double degree $\equiv k+k \equiv 0(\bmod 2)$. This means $\gamma^{k} \in V_{k}$ for every $k \geq 0$. Because $T_{n}(\gamma)$ is $\mathbb{Z}$-linear combination of $\gamma^{k}$ with $k \leq n$, we have $T_{n}(\gamma) \in V_{n}$. The proof for $\bar{\gamma}$ is similar.

Remark 5.4 It is an easy exercise to show that $T_{N}(\bar{\gamma})=\left.T_{N}(\gamma)\right|_{t \rightarrow t^{-1}}$.

\section{Skein module of twice-punctured disk at root of 1}

Recall that $\gamma$ and $\bar{\gamma}$ are knot diagrams on $\mathbb{D}$ defined by (18). The following was proved by Bonahon and Wong, using quantum Teichmüller algebras and their representations.

Proposition 6.1 Suppose $\xi^{4}$ is a root of 1 of order $N$. Then in $\mathcal{S}_{\xi}(\mathbb{D})$ one has

$$
\begin{aligned}
& T_{N}(\gamma)=\xi^{-N^{2}} T_{N}(y)+\xi^{N^{2}} T_{N}\left(x_{1}\right) T_{N}\left(x_{2}\right), \\
& T_{N}(\bar{\gamma})=\xi^{N^{2}} T_{N}(y)+\xi^{-N^{2}} T_{N}\left(x_{1}\right) T_{N}\left(x_{2}\right) .
\end{aligned}
$$

As mentioned above, there was an urge to find a proof using elementary skein theory; one such proof is presented here. Our proof roughly goes as follows. Using the transparent property of $T_{N}(\gamma)$, we show that $T_{N}(\gamma)$ is a common eigenvector of several operators. We then prove that the space of common eigenvectors has dimension at most 3, with a simple basis. We then fix coefficients of $T_{N}(\gamma)$ in this basis using calculations in highest order. Then the result turns out to be the right-hand side of (19).

Throughout this section we fix a complex number $\xi$ such that $\xi^{4}$ is a root of unity of order $N$. Define $\varepsilon=\xi^{N^{2}}$. We will write $V_{N, \xi}$ simply by $V_{N}$ and $\lambda_{k}$ for $\lambda_{k}(\xi)$. Thus, in the whole section,

$$
\lambda_{k}=-\left(\xi^{2 k+2}+\xi^{-2 k-2}\right) .
$$

\subsection{Properties of $\xi$ and $\lambda_{k}$}

Recall that $\xi^{4}$ is a root of 1 of order $N$.

Lemma 6.2 Suppose $1 \leq k \leq N-1$. Then:

(i) $\lambda_{2 k}=\lambda_{0}$ if and only if $k=N-1$.

(ii) $\lambda_{k}=\xi^{2 N} \lambda_{0}$ implies that $k=N-2$. 
(iii) If $N$ is even then $\xi^{2 N}=-1$.

(iv) One has

$$
\xi^{2 N^{2}+2 N}=(-1)^{N+1} .
$$

Proof (i) With $\lambda_{k}=-\left(\xi^{2 k+2}+\xi^{-2 k-2}\right)$, we have

$$
\lambda_{2 k}-\lambda_{0}=-\xi^{-2-4 k}\left(\xi^{4 k}-1\right)\left(\xi^{4 k+4}-1\right) .
$$

Hence, $\lambda_{2 k}-\lambda_{0}=0$ if and only if either $N \mid k$ or $N \mid(k+1)$. With $1 \leq k \leq N-1$, this is equivalent to $k=N-1$.

(ii) We have

$$
\lambda_{k}-\xi^{2 N} \lambda_{0}=-\xi^{-2 N-2}\left(\xi^{2 N-2 k}-1\right)\left(\xi^{2 N+2 k+4}-1\right) .
$$

Either $\xi^{2 N-2 k}=1$ or $\xi^{2 N+2 k+4}=1$. Taking the squares of both identities, we see that either $N \mid(N-k)$ or $N \mid(k+2)$. With $1 \leq k \leq N-1$, we conclude that $k=N-2$.

(iii) Suppose $N$ is even. Since $\xi^{4}$ has order $N$, one has $\left(\xi^{4}\right)^{N / 2}=-1$. Then $\xi^{2 N}=\left(\xi^{4}\right)^{N / 2}=-1$.

(iv) The proof is left for the reader.

\subsection{Operators $\Phi_{i}$ and the vector space $W$}

Recall that $\Phi_{i}:=\Phi_{\alpha_{i}}, i=0,1,2,3$, is defined in Section 5.1. Then $\Phi_{i}\left(V_{N}\right) \subset V_{N}$ for $i=0,1,2,3$.

Let $\Phi_{4}$ be the curve on $\partial \mathbb{D} \times[-1,1]$ depicted in Figure 10. Here we draw $\mathcal{H}=$ $\mathbb{D} \times[-1,1]$ as a handlebody. We also depict $\Phi_{4}\left(x_{2}^{3}\right)$.
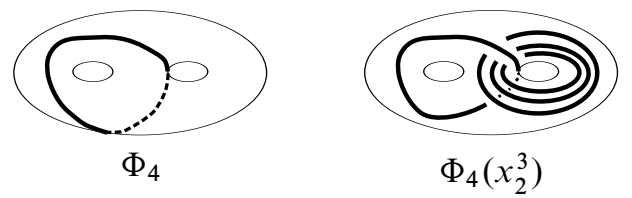

Figure 10: The curve $\Phi_{4}$ and $\Phi_{4}\left(x_{2}^{3}\right)$

We do not have $\Phi_{4}\left(V_{N}\right) \subset V_{N}$, since $\Phi_{4}$ in general increases the double degree. By counting the intersection points with $\alpha_{1}$ and $\alpha_{2}$, we have, for every $E \in \mathcal{S}_{\xi}(\mathbb{D})=$ $\mathbb{C}\left[x_{1}, x_{2}, y\right]$,

$$
\operatorname{deg}_{l r}\left(\Phi_{4}(E)\right) \leq \operatorname{deg}_{l r}(E)+1 .
$$


Proposition 6.3 If $E$ is one of $\left\{T_{N}(\gamma), T_{N}(\bar{\gamma}), T_{N}(y), T_{N}\left(x_{1}\right) T_{N}\left(x_{2}\right)\right\}$, then

$$
\begin{aligned}
\sigma(E) & =E, \\
\Phi_{1}(E) & =\xi^{2 N} \lambda_{0} E, \\
\Phi_{i}(E) & =\lambda_{0} E \quad \text { for } i=0,3, \\
\Phi_{4}(E) & =\xi^{2 N} x_{1} E .
\end{aligned}
$$

Proof The first identity follows from the fact that each of $\gamma, \bar{\gamma}, y, x_{1} \cup x_{2}$ is invariant under $\sigma$. The remaining identities follow from the $\xi^{2 N}$-transparent property of $T_{N}(z)$, Corollary 2.7.

Remark 6.4 Note that $\Phi_{4}$ is $\mathbb{C}\left[x_{1}\right]$-linear and (26) says $E$ is a $\xi^{2 N} x_{1}$-eigenvector of $\Phi_{4}$.

Let $W$ be the subspace of $V_{N}$ consisting of elements satisfying (23)-(26). This means $W \subset V_{N}$ consists of elements which are at the same time 1-eigenvector of $\sigma$, $\xi^{2 N} \lambda_{0}$-eigenvector of $\Phi_{1}, \lambda_{0}$-eigenvector of $\Phi_{0}$ and $\Phi_{3}$, and $\xi^{2 N} x_{1}$-eigenvector of $\Phi_{4}$.

We will show that $W$ is spanned by $T_{N}(y), T_{N}\left(x_{1}\right) T_{N}\left(x_{2}\right)$, and possibly 1 .

\subsection{Action of $\Phi_{3}, \Phi_{0}$ and $\Phi_{1}$}

For an element $F \in \mathbb{C}\left[x_{1}, x_{2}, y\right]$ and a monomial $\mathfrak{m}=x_{1}^{a_{1}} x_{2}^{a_{2}} y^{b}$ let $\operatorname{coeff}(F, \mathfrak{m})$ be the coefficient of $\mathfrak{m}$ in $F$.

Lemma 6.5 Suppose $E \in W$ and $\operatorname{coeff}\left(E, y^{N}\right)=0$. Then $E \in \mathbb{C}\left[x_{1}, x_{2}\right]$.

Proof Let $k$ be the $y$-degree of $E$. Since $E \in W$ and coeff $\left(E, y^{N}\right)=0$, one has $k \leq N-1$.

We need to show that $k=0$. Suppose to the contrary that $1 \leq k$. Then $1 \leq k \leq N-1$.

First we will prove $k=N-1$, using the fact that $E$ is a $\lambda_{0}$-eigenvector of $\Phi_{3}$ by (25).

Recall that $\mathrm{fil}_{\alpha_{3}}$ is twice the $y$-degree. One has $\mathrm{fil}_{\alpha_{3}}(E)=2 k$. Thus $E \neq 0 \in$ $\mathcal{F}_{2 k}^{\alpha_{3}} / \mathcal{F}_{2 k-1}^{\alpha_{3}}$. By Proposition 3.2, any nonzero element in $\mathcal{F}_{2 k}^{\alpha_{3}} / \mathcal{F}_{2 k-1}^{\alpha_{3}}$ is an eigenvector of $\Phi_{3}$ with eigenvalue $\lambda_{2 k}$. But $E$ is an eigenvector of $\Phi_{3}$ with eigenvalue $\lambda_{0}$. It follows that $\lambda_{2 k}=\lambda_{0}$. By Lemma 6.2, we have $k=N-1$.

Because $\operatorname{deg}_{l r}(E)$ is even and less than or equal to $2 N$, we must have

$$
E=y^{N-1}\left(c_{1} x_{1} x_{2}+c_{2}\right)+O\left(y^{N-2}\right), \quad c_{1}, c_{2} \in \mathbb{C} .
$$


We will prove $c_{1}=0$ by showing that otherwise, $\Phi_{0}$ will increase the $y$-degree. Note that $\Phi_{0}$ can increase the $y$-degree by at most 1 , and $\Phi_{0}$ is $\mathbb{C}[y]$-linear. We have

$$
\Phi_{0}(E)=y^{N-1}\left(c_{1} \Phi_{0}\left(x_{1} x_{2}\right)+c_{2} \Phi_{0}(1)\right)+O\left(y^{N-1}\right) .
$$

The diagram of $\Phi_{0}\left(x_{1} x_{2}\right)$ has 4 crossings; see Figure 11.

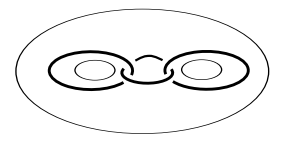

Figure 11: The diagram of $\Phi_{0}\left(x_{1} x_{2}\right)$

A simple calculation shows

$$
\Phi_{0}\left(x_{1} x_{2}\right)=\left(1-t^{4}\right)\left(1-t^{-4}\right) y+O\left(y^{0}\right) .
$$

Plugging this value in (27), with $\Phi_{0}(1)=\lambda_{0} \in \mathbb{C}$,

$$
\Phi_{0}(E)=y^{N} c_{1}\left(1-t^{4}\right)\left(1-t^{-4}\right)+O\left(y^{N-1}\right)
$$

If $c_{1} \neq 0$, then the $y$-degree of $\Phi_{0}(E)$ is $N$, strictly bigger than that of $E$ and $E$ cannot be an eigenvector of $\Phi_{0}$. Thus $c_{1}=0$.

One has now

$$
E=c_{2} y^{N-1}+O\left(y^{N-2}\right) .
$$

Since the $y$-degree of $E$ is $N-1$, one must have $c_{2} \neq 0$. By counting the intersections with $\alpha_{3}$, we see that $\Phi_{1}$ does not increase the $y$-degree. We have

$$
\begin{aligned}
\Phi_{1}(E) & =c_{2} \Phi_{1}\left(y^{N-1}\right)+O\left(y^{N-2}\right) \\
& =c_{2} \lambda_{N-1} y^{N-1}+O\left(y^{N-2}\right) \text { by Proposition 3.2. }
\end{aligned}
$$

Comparing the above identity with (29) and using the fact that $E$ is a $\xi^{2 N} \lambda_{0^{-}}$ eigenvector of $\Phi_{1}$, we have

$$
\lambda_{N-1}=\xi^{2 N} \lambda_{0}
$$

which is impossible since Lemma 6.2 says that $\lambda_{k}=\xi^{2 N} \lambda_{0}$ only when $k=N-2$. This completes the proof of the lemma.

\subsection{Action of $\Phi_{4}$}

Recall that $\Phi_{4}$ is the curve on the boundary of the handlebody $\mathcal{H}$ (see Figure 10) which acts on $\mathcal{S}_{\xi}(\mathbb{D})=\mathbb{C}\left[x_{1}, x_{2}, y\right]$. The action of $\Phi_{4}$ is $\mathbb{C}\left[x_{1}\right]$-linear, and every element of $W$ is a $\xi^{2 N} x_{1}$-eigenvector of $\Phi_{4}$. 
Recall that $\operatorname{deg}_{r}=$ fil $_{\alpha_{2}}$ and $\operatorname{deg}_{r}\left(x_{1}^{a_{1}} x_{2}^{a_{2}} y^{b}\right)=a_{2}+b$. Note that for $F \in \mathbb{C}\left[x_{1}, x_{2}\right]$, $\operatorname{deg}_{r}(F)$ is exactly the $x_{2}$-degree of $F$. By looking at the intersection with $\alpha_{2}$, we see that $\Phi_{4}$ preserves the $\alpha_{2}$-filtration, ie $\operatorname{deg}_{r} \Phi_{4}(F) \leq \operatorname{deg}_{r}(F)$. We will study actions of $\Phi_{4}$ on the associated graded spaces.

We will use the notation $F+\operatorname{deg}_{r}-$ lot to mean $F+F_{1}$, where $\operatorname{deg}_{r}\left(F_{1}\right)<\operatorname{deg}_{r}(F)$.

Lemma 6.6 Suppose $1 \leq k \leq N-1$. One has

$$
\begin{aligned}
\Phi_{4}\left(a\left(x_{1}\right) T_{N}\left(x_{2}\right)\right) & =\xi^{2 N} x_{1}\left[a\left(x_{1}\right) T_{N}\left(x_{2}\right)\right], \\
\Phi_{4}\left(T_{k}\left(x_{2}\right)\right) & =y\left[\xi^{2}\left(\xi^{-2 k}-\xi^{2 k}\right) x_{2}^{k-1}\right]+\operatorname{deg}_{r}-\operatorname{lot}\left(\bmod \mathbb{C}\left[x_{1}, x_{2}\right]\right) .
\end{aligned}
$$

Proof Identity (30) follows from the $\xi^{2 N}$-transparency of $T_{N}(z)$.

Let us prove (31). Applying identity (17) to the dashed box below, we have

$$
\begin{aligned}
\Phi_{4}\left(T_{k}\left(x_{2}\right)\right) & =0 \\
& =y\left[\xi^{2}\left(\xi^{-2 k}-\xi^{2 k}\right) S_{k-1}\left(x_{2}\right)\right]+x_{1}\left[\xi^{-2 k} S_{k}\left(x_{2}\right)-\xi^{2 k} S_{k-2}\left(x_{2}\right)\right],
\end{aligned}
$$

which implies (31).

\subsection{The space $W \cap \mathbb{C}\left[x_{1}, x_{2}\right]$}

Lemma 6.7 Suppose $E \in W \cap \mathbb{C}\left[x_{1}, x_{2}\right]$ and the coefficient of $x_{1}^{N} x_{2}^{N}$ in $E$ is 0 . Then $E \in \mathbb{C}$.

Proof Since $T_{k}\left(x_{2}\right)$ is a basis of $\mathbb{C}\left[x_{2}\right]$, we can write $E$ uniquely as

$$
E=\sum_{k=0}^{N} a_{k}\left(x_{1}\right) T_{k}\left(x_{2}\right), \quad a_{k}\left(x_{1}\right) \in \mathbb{C}\left[x_{1}\right] .
$$

Let $j$ be the $x_{2}$-degree of $E^{\prime}:=E-a_{N}\left(x_{1}\right) T_{N}\left(x_{2}\right)$. Then $j \leq N-1$.

First we will show $j=0$. Assume to the contrary that $j \geq 1$. Thus $1 \leq j \leq$ $N-1$. Note that $E$, by assumption, and $a_{N}\left(x_{1}\right) T_{N}\left(x_{2}\right)$, by (30), are eigenvectors of $\Phi_{4}$ with eigenvalue $\xi^{2 N} x_{1}$. It follows that $E^{\prime}$ is also an eigenvector of $\Phi_{4}$ with eigenvalue $\xi^{2 N} x_{1}$. We have

$$
E^{\prime}=\sum_{k=0}^{j} a_{k}\left(x_{1}\right) \Phi_{4}\left(T_{k}\left(x_{2}\right)\right)=a_{j}\left(x_{1}\right) T_{j}\left(x_{2}\right)+\operatorname{deg}_{r}-\text { lot. }
$$

Using (31) and the fact that $\Phi_{4}$ does not increase $\operatorname{deg}_{r}$, we have

$$
\Phi_{4}\left(E^{\prime}\right)=y\left[a_{j}\left(x_{1}\right) \xi^{2}\left(\xi^{-2 k}-\xi^{2 k}\right) x_{2}^{k-1}\right]+\operatorname{deg}_{r}-\operatorname{lot}\left(\bmod \mathbb{C}\left[x_{1}, x_{2}\right]\right) .
$$


When $1 \leq j \leq n-1$, the coefficient of $y$, which is the element in the square bracket, is nonzero. Thus $\Phi_{4}\left(E^{\prime}\right) \notin \mathbb{C}\left[x_{1}, x_{2}\right]$, while $E^{\prime} \in \mathbb{C}\left[x_{1}, x_{2}\right]$. This means $E^{\prime}$ cannot be an eigenvector of $\Phi_{4}$, a contradiction. This proves $j=0$.

So we have

$$
E=a_{N}\left(x_{1}\right) T_{N}\left(x_{2}\right)+a_{0}\left(x_{1}\right) .
$$

Because $\operatorname{deg}_{l r}(E)<2 N$, the $x_{1}$-degree of $a_{N}\left(x_{1}\right)$ is less than $n$. Using the invariance under $\sigma$, one sees that $E$ must be of the form

$$
E=c_{1}\left(T_{N}\left(x_{1}\right)+T_{N}\left(x_{2}\right)\right)+c_{2}, \quad c_{1}, c_{2} \in \mathbb{C} .
$$

To finish the proof of the lemma, we need to show that $c_{1}=0$. Assume that $c_{1} \neq 0$. Since $E$ has even double degree, $N$ is even. By Lemma 6.2(iii), $\xi^{2 N}=-1$.

Recall that $E$ is a $\lambda_{0}$-eigenvector of $\Phi_{0}$. Applying $\Phi_{0}$ to (32),

$$
\lambda_{0}\left[c_{1}\left(T_{N}\left(x_{1}\right)+T_{N}\left(x_{2}\right)\right)+c_{2}\right]=\Phi_{0}\left(c_{1}\left(T_{N}\left(x_{1}\right)+T_{N}\left(x_{2}\right)\right)+c_{2}\right) .
$$

Both $T_{N}\left(x_{1}\right)$ and $T_{N}\left(x_{2}\right)$ are eigenvectors of $\Phi_{0}$ with eigenvalues $\xi^{2 N} \lambda_{0}=-\lambda_{0}$, while $\Phi_{0}(1)=\lambda_{0}$. Hence we have

$$
\lambda_{0}\left[c_{1}\left(T_{N}\left(x_{1}\right)+T_{N}\left(x_{2}\right)\right)+c_{2}\right]=\lambda_{0}\left[-c_{1}\left(T_{N}\left(x_{1}\right)-T_{N}\left(x_{2}\right)\right)+c_{2}\right],
$$

which is impossible since $c_{1} \lambda_{0} \neq 0$. Hence, we have $c_{1}=0$ and $E \in \mathbb{C}$.

\subsection{Some maximal degree parts of $T_{N}(\gamma)$}

Lemma 6.8 One has

$$
\begin{aligned}
\operatorname{coeff}\left(T_{N}(\gamma), y^{N}\right) & =\xi^{-N^{2}}, \\
\operatorname{coeff}\left(T_{N}(\gamma), x_{1}^{N} x_{2}^{N}\right) & =\xi^{N^{2}} .
\end{aligned}
$$

Proof Since $T_{N}(\gamma)=\gamma^{N}+\operatorname{deg}_{l r}-$ lot, we have $\operatorname{coeff}\left(T_{N}(\gamma), y^{N}\right)=\operatorname{coeff}\left(\gamma^{N}, y^{N}\right), \quad \operatorname{coeff}\left(T_{N}(\gamma), x_{1}^{N} x_{2}^{N}\right)=\operatorname{coeff}\left(\gamma^{N}, x_{1}^{N} x_{2}^{N}\right)$.

There are $N^{2}$ crossing points in the diagram of $\gamma^{N}$. Each crossing can be smoothed in two ways. The positive smoothing acquires a factor $t$ in the skein relation, and the negative smoothing acquires a factor $t^{-1}$. The are $2^{N^{2}}$ smoothings of $\gamma^{N}$. Each smoothing $s$ of all the $N^{2}$ crossings gives rise to a link $L_{s}$ embedded in $\mathbb{D}$. Then $\gamma^{N}$ is a linear combination of all $L_{s}$. We will show that the only $s$ for which $L_{s}=y^{N}$ is the all negative smoothing.

Consider a crossing point $C$ of $\gamma^{N}$. The vertical line passing through $C$ intersects $\mathbb{D}$ in an interval $\alpha_{3}^{\prime}$ which is isotopic to $\alpha_{3}$, and fil $\alpha_{3}=\mathrm{fil}_{\alpha_{3}^{\prime}}$. For an embedded link $L$ in $\mathbb{D}$, 
as an element of $\mathcal{S}(\mathbb{D})=R\left[x_{1}, x_{2}, y\right], L$ is a monomial whose $y$-degree is bounded above by half the number of intersection points of $L$ with $\alpha_{3}^{\prime}$. The diagram $\gamma^{N}$ has exactly $2 N$ intersection points with $\alpha_{3}^{\prime}$, with $C$ contributing two (of the $2 N$ intersection points). If we positively smooth $\gamma^{N}$ at $C$, the result is a link diagram with $2 N-2$ intersection points with $\alpha_{3}^{\prime}$, and no matter how we smooth other crossings, the resulting link will have less than or equal to $2 N-2$ intersection points with $\alpha_{3}^{\prime}$. Thus we cannot get $y^{N}$ if any of the crossing is smoothed positively. The only smoothing which results in $y^{N}$ is the all negative smoothing. The coefficient of this smoothing is $\xi^{-N^{2}}$.

Similarly, one can prove that the only smoothing which results in $x_{1}^{N} x_{2}^{N}$ is the all positive smoothing, whose coefficient is $\xi^{N^{2}}$.

\subsection{Proof of Proposition 6.1}

Let

$$
E=T_{N}(\gamma)-\xi^{N^{2}} T_{N}\left(x_{1}\right) T_{N}\left(x_{2}\right)-\xi^{-N^{2}} T_{N}(y) .
$$

Then $E \in W$. Lemma 6.8 shows that $\operatorname{coeff}\left(E, y^{N}\right)=0=\operatorname{coeff}\left(E, x_{1}^{N} x_{2}^{N}\right)$. By Lemma 6.5, $E \in \mathbb{C}\left[x_{1}, x_{2}\right]$. Then by Lemma 6.7, we have $E \in \mathbb{C}$, ie $E$ is a constant.

We will show that $E=0$. This is done by using the inclusion of $\mathcal{H}$ into $\mathbb{R}^{3}$, which gives a $\mathbb{C}$-linear map $\iota: \mathcal{S}_{\xi}(\mathbb{D}) \rightarrow \mathcal{S}_{\xi}\left(\mathbb{R}^{3}\right)=\mathbb{C}$. Under $\iota$, we have

$$
E=\iota\left(T_{N}(\gamma)\right)-\iota\left(\xi^{N^{2}} T_{N}\left(x_{1}\right) T_{N}\left(x_{2}\right)\right)-\iota\left(\xi^{-N^{2}} T_{N}(y)\right) .
$$

The right-hand side involves the trivial knot and the trivial knot with framing 1 , and can be calculated explicitly as follows. Note that $\iota(\gamma)$ is the unknot with framing 1, while $\iota\left(x_{1}\right)=\iota\left(x_{2}\right)=\iota(y)=U$, the trivial knot. With $T_{N}=S_{N}-S_{N-2}$, and the framing change given by (14), we find

$$
T_{N}(\mathrm{l})=(-1)^{N} \xi^{N^{2}+2 N} T_{N}(\mid)=-\xi^{-N^{2}} T_{N}(\mid),
$$

where the second identity follows from (21). Similarly, using (13), we have

$$
T_{N}(L \sqcup U)=2(-1)^{N} \xi^{2 N} T_{N}(L)=-\left(\xi^{2 N^{2}}+\xi^{-2 N^{2}}\right) T_{N}(L) .
$$

From (36) and (37), we calculate the right-hand side of (35), and find that $E=0$. This proves (19).

The proof of (20) is similar. Alternatively, one can get (20) from (19) by noticing that the mirror image map on $R\left[x_{1}, x_{2}, y\right]$ is the $\mathbb{C}$-algebra map sending $t$ to $t^{-1}$, leaving each of $x_{1}, x_{2}, y$ fixed.

This completes the proof of Proposition 6.1. 


\section{Proof of Theorem 2}

Recall that $\varepsilon=\xi^{N^{2}}$, where $\xi^{4}$ is a root of 1 of order $N$. Then $\varepsilon^{4}=1$. The map $\mathcal{S}_{\varepsilon}(M) \rightarrow \mathcal{S}_{\xi}(M)$, defined for framed links by $L \rightarrow T_{N}(L)$, is well defined if and only if it preserves the skein relations (1) and (2), ie in $\mathcal{S}_{\xi}(M)$,

$$
\begin{aligned}
T_{N}(L) & =\varepsilon T_{N}\left(L_{+}\right)+\varepsilon^{-1} T_{N}\left(L_{-}\right), \\
T_{N}(L \sqcup U) & =-\left(\varepsilon^{2}+\varepsilon^{-2}\right) T_{N}(L) .
\end{aligned}
$$

Here, in (38), $L, L_{+}, L_{-}$are links appearing in the original skein relation (1), they are identical everywhere, except in a ball $B$, where they appear as in Figure 12.

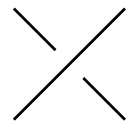

$L$

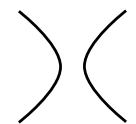

$L_{+}$

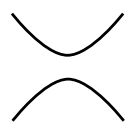

$L_{-}$

Figure 12: The links $L, L_{+}$and $L_{-}$

Identity (39) follows from (37). Let us prove (38).

Case 1 The two strands of $L$ in the ball $B$ belong to the same component. Then (38) follows from Proposition 6.1, applied to the handlebody which is the union of $B$ and a tubular neighborhood of $L$.

Case 2 The two strands of $L$ in $B$ belong to different components. Then the two strands of $L_{+}$belong to the same component, and we can apply (38) to the case when the left-hand side is $L_{+}$. We have

$$
\begin{aligned}
& T_{N}\left(L_{+}\right)=T_{N}()()=T_{N}(\text { D }) \\
& =\varepsilon^{-1} T_{N}(\lambda)+\varepsilon T_{N}(\text { R }) \\
& =\varepsilon^{-1} T_{N}(\mathcal{S})+\varepsilon(-\varepsilon) T_{N}(\asymp) \\
& =\varepsilon^{-1} T_{N}(L)-\varepsilon^{2} T_{N}\left(L_{-}\right),
\end{aligned}
$$

where (40) follows from Case 1 and (41) follows from the framing factor formula (36). Multiplying (42) by $\varepsilon$ and using $\varepsilon^{3}=\varepsilon^{-1}$, we get (38) in this case. This completes the proof of Theorem 2 .

Remark 7.1 In [2], in order to prove Theorem 2, the authors proved in addition to Proposition 6.1 a similar statement for links in the cylinder over a punctured torus. 
Here we bypass this extra statement by reducing the extra statement to Proposition 6.1. Essentially this is due to the fact that the cylinder over a punctured torus is the same as the cylinder over a twice-punctured disk.

\section{References}

[1] C Blanchet, N Habegger, G Masbaum, P Vogel, Three-manifold invariants derived from the Kauffman bracket, Topology 31 (1992) 685-699 MR1191373

[2] F Bonahon, H Wong, Representations of the Kauffman skein algebra I: Invariants and miraculous cancellations arXiv:1206.1638

[3] F Bonahon, H Wong, Quantum traces for representations of surface groups in $\mathrm{SL}_{2}(\mathbb{C})$, Geom. Topol. 15 (2011) 1569-1615 MR2851072

[4] D Bullock, Rings of $\mathrm{SL}_{2}(\mathbb{C})$-characters and the Kauffman bracket skein module, Comment. Math. Helv. 72 (1997) 521-542 MR1600138

[5] D Bullock, C Frohman, J Kania-Bartoszyńska, Understanding the Kauffman bracket skein module, J. Knot Theory Ramifications 8 (1999) 265-277 MR1691437

[6] D Bullock, J H Przytycki, Multiplicative structure of Kauffman bracket skein module quantizations, Proc. Amer. Math. Soc. 128 (2000) 923-931 MR1625701

[7] V V Fok, L O Chekhov, Quantum Teichmüller spaces, Teoret. Mat. Fiz. 120 (1999) 511-528 MR1737362 In Russian; translated in Theoret. and Math. Phys. 110 (1999) $1245-1259$

[8] C Frohman, R Gelca, Skein modules and the noncommutative torus, Trans. Amer. Math. Soc. 352 (2000) 4877-4888 MR1675190

[9] R M Kashaev, Quantization of Teichmüller spaces and the quantum dilogarithm, Lett. Math. Phys. 43 (1998) 105-115 MR1607296

[10] L H Kauffman, State models and the Jones polynomial, Topology 26 (1987) 395-407 MR899057

[11] T T Q Lêe, The colored Jones polynomial and the A-polynomial of knots, Adv. Math. 207 (2006) 782-804 MR2271986

[12] T T Q Lê, A T Tran, The Kauffman bracket skein module of two-bridge links, Proc. Amer. Math. Soc. 142 (2014) 1045-1056 MR3148538

[13] W B R Lickorish, An introduction to knot theory, Graduate Texts in Math. 175, Springer (1997) MR1472978

[14] J Marché, The skein module of torus knots, Quantum Topol. 1 (2010) 413-421 MR2733247

[15] J H Przytycki, Fundamentals of Kauffman bracket skein modules, Kobe J. Math. 16 (1999) 45-66 MR1723531 
[16] J H Przytycki, A S Sikora, On skein algebras and $\mathrm{SL}_{2}(\mathbb{C})$-character varieties, Topology 39 (2000) 115-148 MR1710996

[17] A S Sikora, Skein modules at the $4^{\text {th }}$ roots of unity, J. Knot Theory Ramifications 13 (2004) 571-585 MR2080123

[18] V G Turaev, Skein quantization of Poisson algebras of loops on surfaces, Ann. Sci. École Norm. Sup. 24 (1991) 635-704 MR1142906

School of Mathematics, Georgia Institute of Technology

686 Cherry Street, Atlanta, GA 30332, USA

letu@math.gatech.edu

Received: 24 May 2014 Revised: 1 September 2014 
